\title{
Optical Trocar Causing Aortic Injury: A Potentially Fatal Complication of Minimal Access Surgery
}

\author{
Rashid Usman1, Hafsa Ahmed1, Zeeshan Ahmed1 and Maqsood Ali2 \\ Department of Surgery1 / Anesthesia², Mid City Hospital, Lahore, Pakistan
}

\begin{abstract}
Trocar injury to abdominal aorta is uncommon and even rare with optical trocars. Such injury, resulting from umbilical trocar insertion, is potentially fatal. It often causes on-table death due to torrential life-threatening haemorrhage and unavailability of expert vascular help. We present a rare case of an injury to infra-renal abdominal aorta, caused by optical trocar insertion for bariatric surgery. Immediate recognition of the injury, deployment of life-saving manoeuvres, timely resuscitation, followed by definitive repair of aorta by vascular surgeon was life-saving for this patient. The recovery phase was uneventful and patient had no residual clinical problems during follow-up.
\end{abstract}

Key Words: Aorta injury, Laparoscopy, Optical trocar, Bariatric.

How to cite this article: Usman R, Ahmed H, Ahmed Z, Ali M. Optical trocar causing aortic injury: A potentially fatal complication of minimal access surgery. J Coll Physicians Surg Pak 2020; 30(1):85-87.

\section{INTRODUCTION}

Metabolic surgery is steadily increasing in demand with the diagnosis of metabolic syndrome. Surgeons and patients both prefer laparoscopic approach due to its decreased morbidity and early recovery. ${ }^{1}$ Although visceral injuries are reported but the incidence of injury to the retroperitoneal major vessels, as a result of first trocar insertion, is only $0.02 \% .^{2}$ The chances of such injuries are further reduced with the use of optical trocars; however, the rare chance of injury to aorta still exists even with all these modern precautions. Such injury results in immediate major hemodynamic collapse; hence, early detection and immediate explorative laparotomy may prove life-saving.

We present management of a rare case of infra-renal abdominal aortic injury occurring with an optical trocar, in a patient undergoing an elective sleeve gastrectomy.

\section{CASE REPORT}

A 36-year lady with no comorbidities was scheduled for elective laparoscopic sleeve gastrectomy under general anaesthesia. Her blood pressure was $110 / 75 \mathrm{mmHg}$ and pulse was 85 beats per minute at the time of umbilical trocar insertion. While the first optical trocar (VisiportTM Plus $12 \mathrm{~mm}$ diameter, $15 \mathrm{~cm}$ length) was being inserted to gain abdominal access, the sudden forceful push was

Correspondence to: Dr. Rashid Usman, Department of Surgery, Mid City Hospital and Combined Military Hospital, Lahore,

Pakistan

E-mail:drrashidusman@yahoo.com

Received: January 22, 2019; Revised: March 04, 2019;

Accepted: April 20, 2019 followed by immediate hemodynamic collapse and cardiac arrest. The pneumoperitoneum had not yet been created and blood at the port site was noted. The patient was defibrillated and fluid resuscitation was started. Meanwhile, immediate midline laparotomy revealed a large expanding retroperitoneal hematoma extending into the pelvis. Retroperitoneum was incised just to the left of root of mesentery. Evacuation of hematoma revealed an injury to the antero-lateral wall of the aorta, approximately $4 \mathrm{~cm}$ proximal to the aortic bifurcation. Immediate digital pressure was applied to the bleeding point and patient was hemodynamically resuscitated; while an emergency call for a vascular surgeon was sent. A sinus rhythm with tachycardia and a recordable systolic blood pressure of $80 \mathrm{mmHg}$ was obtained. The vascular surgeon exposed the aorta proximally up to the origin of the renal vessels and distally up to the

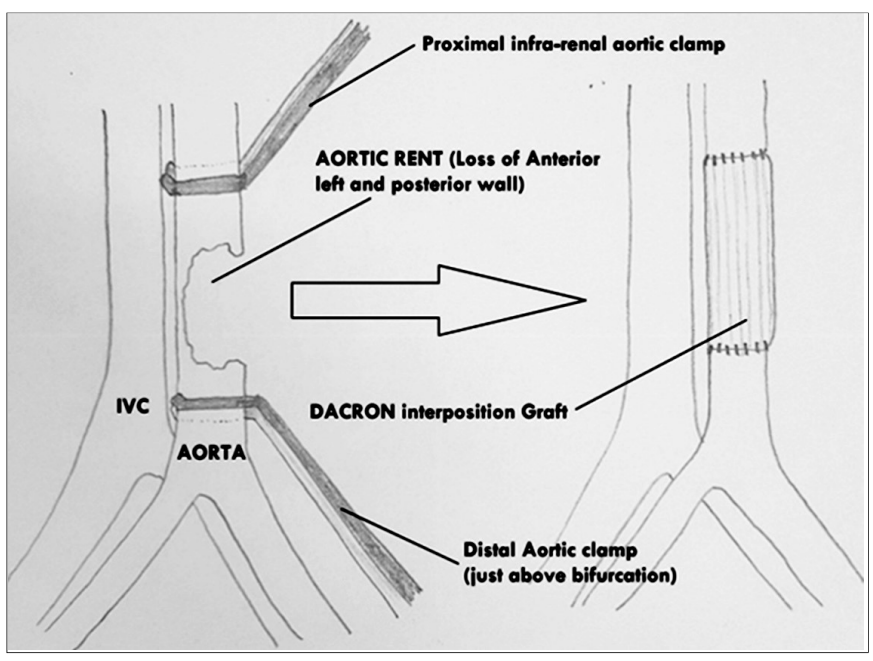

Figure 1: Schema of the aortic injury and its repair. 


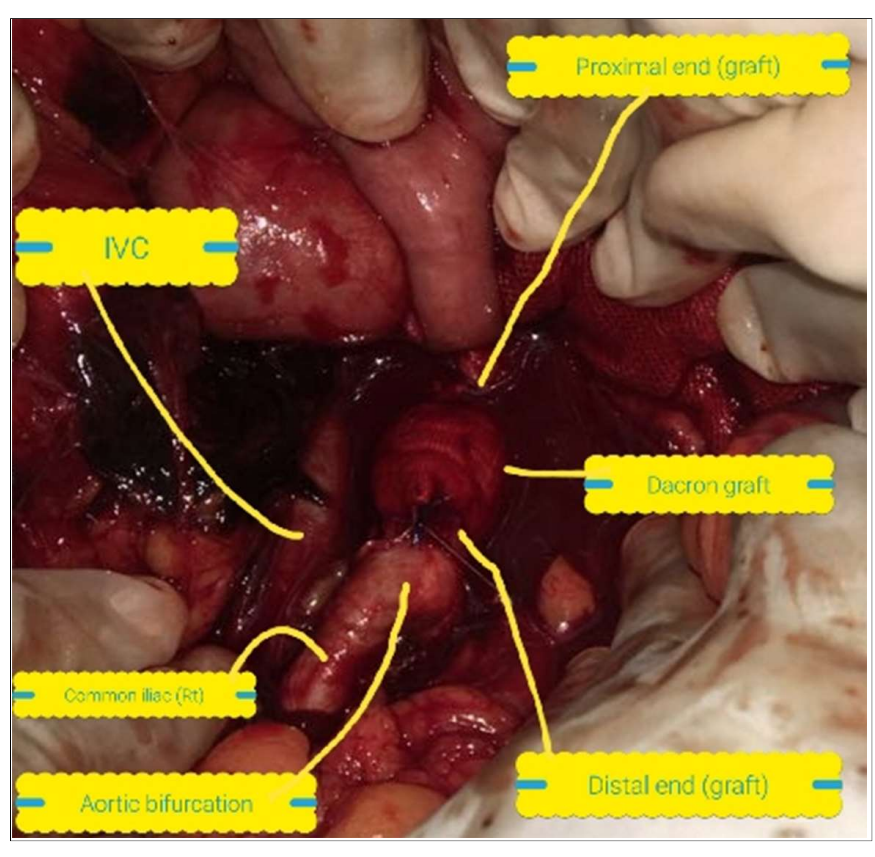

Figure 2: Perioperative photograph after repair with Dacron graft.

bifurcation. On the right, the inferior vena cava was also exposed adjacent to the injury site to assess any concomitant venous injury; however, none was found. The macerated margins of the injured aorta were debrided. After the debridement, the defect was approximately $3 \mathrm{~cm}$ in cranio-caudal direction and involved the whole anterior and postero-lateral wall on the left side of the vessel (Figure 1). Considering the size and extent of the defect, primary or patch repair was not considered. The aorta was completely transected and a polyester woven graft $(14 \mathrm{~mm}$ diameter and $4 \mathrm{~cm}$ length) was interposed between the divided aortic segments (Figure 2). During the repair, permissive hypotension was maintained. The total aortic clamp time was approximately one hour. The patient received 8 units of red cell concentrate and 6 units of fresh frozen plasma in addition to the colloids and crystalloids during the surgery. She was kept on elective ventilatory support in Intensive Care Unit postoperatively and was weaned off the ventilator after 24 hours. The patient recovered steadily without any postoperative complication, and was discharged on her $8^{\text {th }}$ postoperative day.

\section{DISCUSSION}

Obesity is coming forward as an epidemic leading to ill health. ${ }^{3}$ With increased awareness of complications of obesity, the demand for metabolic surgery has also expanded. Laparoscopic approach in bariatric surgery is preferred by the patient as well as the physician, considering its obvious advantages.

Establishment of pneumoperitoneum in an obese patient is one of the most technically demanding steps, with majority vascular injuries occurring during it. Open and closed techniques are employed for creating pneumoperitoneum. The closed techniques are either the use of Veress needle or direct trocar insertion (DTI). According to a research, it is estimated that $40 \%$ of surgeons use the Veress needle, $30 \%$ use DTI and $30 \%$ use the open technique. ${ }^{4}$ Sasmal et al. concluded that $0.14 \%$ cases had major vascular injuries when DTI was used. $5 \mathrm{~A}$ recent meta-analysis concluded that DTI causes fewer major vascular injuries when compared with other two techniques; however, the difference was statistically not significant. 6 In this case, DTI was employed; however, it was with an optical trocar which theoretically should reduce further the incidence of major vascular injury. Lack of experience of surgeon, use of too much force, U-shaped vigorous motion technique, thin linea alba, abnormal angulation, and inappropriate length of trocar are all the risk factors for injuring a major vessel while inserting a primary trocar. ${ }^{7}$

Identification of the injury is the most critical factor effecting the outcome. Damage control by immediate exploration and point pressure on bleeding aorta are lifesaving manoeuvres. In this case, the injury was picked up immediately by the team, since it led to instant hemodynamic instability. The decision by the surgeon to convert to a laparotomy, requesting the help of a vascular surgeon and aggressive resuscitation by the anaesthetist, all contributed to the survival of this patient.

latrogenic aortic injury is generally repaired by simple direct suture or by endovascular technique by stent grafting. 6,7 However, in this patient, the defect size was large and was not suitable for direct repair since it involved loss of more than $2 / 3$ rd of circumference, a woven interposition graft was used. We did not have the facilities for endovascular repair in our setup. This experience taught us that no surgery should be deemed too easy or routine. Injuries can occur even in the most experienced surgical hands; and it takes team effort to respond and save the life of the patient.

Major aortic injury is a rare, but a potentially fatal complication of umbilical trocar insertion. Lack of immediate vascular expertise should not delay life-saving maneuvers, such as point pressure at bleeding aorta along with aggressive volume resuscitation.

\section{CONFLICT OF INTEREST:}

Authors declared no conflict of interest.

\section{AUTHORS' CONTRIBUTION:}

$\mathrm{RU}$ : Study conception; manuscript writing; critical review and revision; final approval of the article.

HA: Study conception; data collection; critical review and revision; final approval of the article.

ZA: Analysis; investigation; critical review and revision; final approval of the article.

MA: Analysis; investigation; manuscript writing; critical review and revision; final approval of the article. 


\section{REFERENCES}

1. McGuire MM, Nadler EP, Qureshi FG. Laparoscopic vertical sleeve gastrectomy for adolescents with morbid obesity. Semin Pediatr Surg 2014; 23:21-3.

2. Asfour V, Smythe E, Attia R. Vascular injury at laproscopy: A guide to management. J Obstet Gynaecol 2018; 38:598-606.

3. Corey KE, Kaplan LM. Obesity and liver disease: The epidemic of twenty-first century. Clin Liver Dis 2014; 18:1-18.

4. Merlin TL, Hiller JE, Maddern GJ, Jamieson GG, Brown AR, Kolbe A. Systematic review of the safety and effectiveness of methods used to establish pneumoperitoneum in laparoscopic surgery. Br J Surg 2003; 90:668-9.
5. Sasmal PK, Tantia O, Jain M, Khanna S, Sen B. Primary access-related complications in laparoscopic cholecystectomy via the closed technique: Experience of a single surgical team over more than 15 years. Surg Endosc 2009; 23: 2407-15.

6. Nishimura M, Matsumoto S, Ohara Y, Minowa K, Tsunematsu R, Takimoto $\mathrm{K}$, et al. Complications related to the initial trocar insertion of 3 different techniques: A systematic review and meta-analysis. J Minim Invasive Gynecol 2019; 26: 63-70.

7. Pring CM. Aortic injury using the hasson trocar: A case report and review of the literature. Ann R Coll Surg Engl 2007; 89: W3-W5. 